\title{
HIGHLIGHTS
}

Treassharraron

\section{Integrative peptidomics identifies acute renal-transplant rejection biomarkers}

The detection of collagen-degradation products in urine may enable the noninvasive diagnosis of acute rejection in renal transplant recipients, according to new findings. The researchers of this study hope that their findings will help elucidate the relationship between urine peptide biomarkers and allograft proteolytic networks that are activated in acute rejection.

Xuefeng Ling, Tara Sigdel and colleagues performed peptidomic analyses on urine samples from 50 renal transplant recipients and 20 nontransplanted controls. The renal transplant recipients had either biopsy-proven acute allograft rejection $(n=20)$, a stable allograft $(n=20)$, or BK-virus nephropathy $(n=10)$. The researchers used liquid chromatography and mass spectrometry to identify 53 candidate peptide peaks that separated transplant recipients with biopsy-proven acute rejection from the other patients. The 53 peptide peaks were then mapped to
40 unique peptides, which the researchers then grouped on the basis of their common protein precursor. The peptides mapped to nine different proteins - uromodulin and eight proteins from the collagen familyall of which were expressed at lower levels in the urine of patients with acute rejection than other patients.

To investigate the relationship between the candidate urinary biomarkers and the rejection process, the researchers analyzed microarray data from matched kidney-biopsy samples. Consistent with the low level of uromodulin in the urine of patients with acute rejection, expression levels of the corresponding gene were lower in biopsy samples from patients with acute rejection than in samples from other patients. By contrast, levels of three collagen genes were upregulated in biopsy samples from patients with acute rejection.

The researchers say that their findings indicate that the dysregulation of collagen

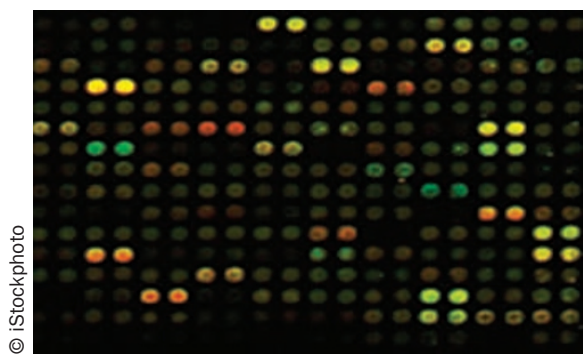

expression between the rejecting graft and urine may provide insight into the mechanisms of acute rejection. They also believe that increased turnover of renal collagens may be a valuable biomarker for the noninvasive diagnosis of acute rejection after renal transplantation.

Susan J. Allison

Original article Ling, X. B. et al. Integrative urinary peptidomics in renal transplantation identifies biomarkers for acute rejection. J. Am Soc. Nephrol. 21, 646-653 (2010) 